\title{
Small bowel gas in severe ulcerative colitis
}

\author{
C N Chew, D J Nolan, D P Jewell
}

\begin{abstract}
The prognostic significance of excess small bowel gas on a plain abdominal radiograph has been assessed in 75 patients with severe attacks of ulcerative colitis requiring intravenous hydrocortisone. The radiographs were reviewed without knowledge of the subsequent outcome. Small bowel distension was defined as the presence of three or more loops of gas filled small bowel. Forty two patients responded to medical treatment and 33 underwent colectomy. The two groups were comparable for age, sex, and length of history. The surgical group had more extensive disease. Of those who did well on medical therapy, 18 (42.9\%) had small bowel distension compared with 24 of $33(72.7 \%)$ who failed medical therapy. The difference was significant $(p<0.05$, odds ratio $=3.55,95 \%$ confidence interval of $2 \cdot 27-5 \cdot 87$ ). Of the 24 patients with small bowel distension who came to surgery, five had more than four loops of gas filled small bowel. This degree of distension was not seen in any of the patients settling on medical therapy. Thus the presence of small bowel distension on a plain abdominal radiograph in a patient with severe ulcerative colitis may predict a poor response to medical therapy.
\end{abstract}

The plain radiograph of the abdomen is usually the first radiological examination in patients with severe ulcerative colitis. It may reveal the presence of complications such as toxic megacolon or perforation as well as extra intestinal manifestations such as sacroiliitis.

Patients with severe ulcerative colitis complicated by toxic megacolon not infrequently have large amounts of gas in the stomach and small bowel. ${ }^{1-3}$ It has been suggested that abnormal small bowel distension may identify a subgroup of patients with severe ulcerative colitis who are particularly prone to the development of toxic megacolon. ${ }^{+5}$ We reviewed the radiographs of 75 patients with severe ulcerative colitis to determine the association of abnormal small bowel distension with subsequent outcome.

\section{Methods}

PATIENTS

Gastroenterology Unit and Department of Radiology, John Radcliffe Hospital, Headington, Oxford

C N Chew

D P Jewell

D J Nolan

Correspondence to:

Dr D P Jewell.

Accepted for publication

ll February 1991
The medical records and radiographs of 75 patients with severe ulcerative colitis admitted to the Gastroenterology Unit of John Radcliffe Hospital between 1985 and 1990 were studied. Disease activity was defined by the criteria of Truelove and Witts. ${ }^{6}$

All patients had been admitted for intravenous corticosteroid therapy. Patients had nil by mouth except water and were given intravenous fluids, intravenous hydrocortisone (100 mg qid) and hydrocortisone enemas (100 $\mathrm{mg}$ bd) for five to seven days. The diagnosis of ulcerative colitis was based on clinical, radiological, endoscopic, and histological findings. Patients were seen daily by the physician (DPJ) in conjunction with one or other of two surgical teams with a special interest in inflammatory bowel disease.

The plain abdominal radiographs of these 75 patients were reviewed retrospectively by two observers, one being a consultant radiologist. The observers were blind to the subsequent clinical outcome of the patients. Plain radiographs of the abdomen were carried out on the day of admission and were repeated every one to three days, depending upon the progress of the patients. Full blood counts and plasma electrolytes were measured daily and the erythrocyte sedimentation rate (ESR) was obtained initially and at five days.

\section{CRITERIA FOR RADIOLOGICAL DIAGNOSES}

Small bowel distension was defined as the presence of three or more loops of gas filled small bowel on a plain abdominal radiograph (Fig 1). Small bowel dilatation was said to be present when the transverse diameter of the small bowel was greater than $3 \mathrm{~cm}$. The extent of colonic disease was estimated by the criteria of Bartram, - namely, the extent of faecal residue, evidence

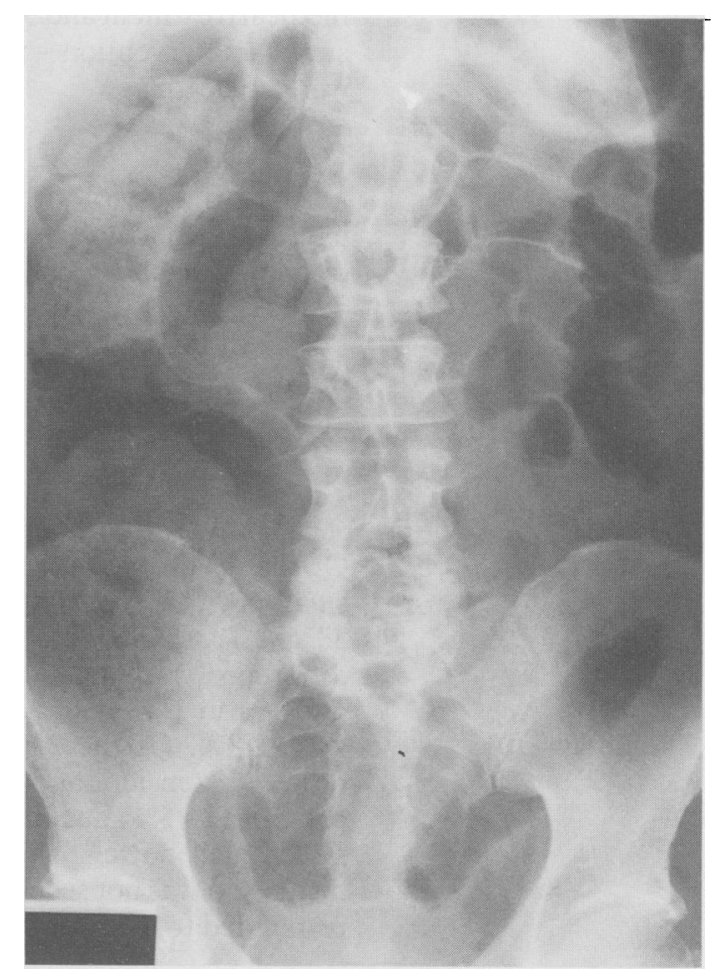

Figure: Plain abdominal radiograph of a patient with severe ulcerative colitis involving the distal colon showing small bowel distension. 
Patients with severe attacks of ulcerative colitis - responders to medical therapy and those that required surgery

\begin{tabular}{lll}
\hline & Medical therapy & Surgery \\
\hline Patients (n) & 42 & 33 \\
Males & $17(40 \cdot 5 \%)$ & $20(60 \cdot 6 \%)$ \\
Females & $25(59 \cdot 5 \%)$ & $13(39 \cdot 4 \%)$ \\
Mean age (SD) yr & $42 \cdot 4(16 \cdot 8)$ & $42 \cdot 8(18)$ \\
Duration of disease (yr) & $11(26 \cdot 2 \%)$ & $7(21 \cdot 2 \%)$ \\
<One & $15(35 \cdot 7 \%)$ & $10(30 \cdot 3 \%)$ \\
$1-5$ & $4(9 \cdot 5 \%)$ & $6(18 \cdot 2 \%)$ \\
$6-10$ & $12(28 \cdot 6 \%)$ & $10(30 \cdot 3 \%)$ \\
$>10$ & $7(8 \cdot 5)$ & $8 \cdot 9(8 \cdot 9)$ \\
Mean (SD) & & \\
\hline
\end{tabular}

of mucosal ulceration and alteration of haustral pattern. The presence of mucosal islands was also noted. Toxic megacolon was defined as an inflamed transverse colon greater than $5.5 \mathrm{~cm}$ in diameter. ${ }^{8}$

\section{ASSESSMENT OF OUTCOME}

The clinical outcomes of the severe attack were remission, the occurrence of major complications, and surgical intervention.

\section{STATISTICAL ANALYSIS}

Results are presented as mean (SD) unless otherwise stated. The data were subjected to $\chi^{2}-$ test, Student's $t$ test and Wilcoxon's rank-sum test where appropriate. Statistical significance between groups was considered to be present when $\mathrm{p}<0.05$.

\section{Results}

Seventy five patients ( 37 male and 38 female) were studied, all had severe ulcerative colitis. Forty two $(56 \%)$ of these patients settled with medical treatment and $33(44 \%)$ patients required surgical intervention - that is, colectomy. There were no deaths.

AGE, SEX, DURATION OF ULCERATIVE COLITIS

The two groups were comparable for age, sex distribution and the length of history of the disease (Table). The mean age of the patients in the study was 43 years $(15-80 \mathrm{yr})$. The mean duration of disease was 7.8 years (one week to $28 \mathrm{yr}$ ).

\section{SMALL BOWEL DISTENSION}

Of the 42 on medical therapy, $18(42.9 \%)$ had small bowel distension compared with 24 of the $33(72 \cdot 7 \%)$ who failed medical therapy. This difference was statistically significant $(p<0.05$, odds ratio (OR) $3 \cdot 55,95 \%$ confidence limits $2 \cdot 27-5 \cdot 87$ ). Of the 24 patients who had small bowel distension and went for surgery, five patients $(24.5 \%)$ had more than four loops of gas filled small bowel. This degree of small bowel distension was not seen in any of the patients that responded to medical therapy. medical treatment had small bowel dilatation on the plain abdominal radiographs compared with 10 of the $33(30 \cdot 3 \%)$ who came to colectomy. Although there was an obvious trend in favour of the poor prognostic group, the difference was not statistically significant.

\section{EXTENT OF ULCERATIVE COLITIS}

Of the group on medical treatment six (14.3\%) patients had pancolitis and 36 had less extensive disease. In contrast 17 of the $33(51.5 \%)$ patients who came to colectomy had pancolitis and 16 patients had less extensive disease. The patients with pancolitis were more likely to come to surgery when compared with those with less extensive involvement of the colon $(\mathrm{p}<0.005$, $\mathrm{OR}=6 \cdot 38,95 \%$ confidence limits $2 \cdot 06-19 \cdot 76$ ).

The extent of the ulcerative colitis did not influence the likelihood of finding moderate small bowel distension on the plain abdominal radiographs of these patients.

\section{MUCOSAL ISLANDS ON THE PLAIN ABDOMINAL} FILMS

Of the 75 patients, 10 had mucosal islands on the plain abdominal radiographs and eight of these 10 patients required colectomy. These eight patients also showed small bowel distension on the plain films. Two patients with mucosal islands had attacks of severe ulcerative colitis which settled with medical treatment. One of these had evidence of small bowel distension on the plain abdominal radiograph.

\section{LABORATORY DATA}

The ESR patients who responded to medical treatment (median $23 \mathrm{~mm}(2-110 \mathrm{~mm})$ ) was significantly lower $(\mathrm{p}<0.05$, Wilcoxon's ranksum test) than that of those who required surgical intervention (median $51 \mathrm{~mm}$, (5-140 mm)).

The serum potassium concentrations measured on the day the radiographs were carried out were noted. There was no significant difference in the serum potassium concentration between the group with small bowel distension (mean serum potassium $3.85(0.47) \mathrm{mmol} / \mathrm{l})$ and the group without distension $(3.77(0.4) \mathrm{mmol} / \mathrm{l})$. There was also no difference in the serum potassium between those that required surgical intervention and those that responded to medical therapy $(3.9(0.47) \mathrm{mmol} / \mathrm{l}$ and $3.74(0.42)$ $\mathrm{mmol} / \mathrm{l}$ respectively).

\section{COMPLICATIONS}

\section{Toxic megacolon}

Only three patients developed toxic megacolon. There was no definite pattern of small bowel gaseous distension or dilatation present in these patients. One had no evidence of small bowel distension or dilatation on the plain abdominal radiograph, another had small bowel distension as well as dilatation, and the third patient had small bowel distension without evidence of small bowel dilatation. 


\section{Massive haemorrhage}

One patient underwent colectomy for massive bleeding from a severely ulcerated descending colon. The plain abdominal radiograph showed no evidence of small distension or dilatation.

\section{Discussion}

Plain radiological films of the abdomen are usually undertaken in the initial assessment of patients with severe attacks of ulcerative colitis. It is useful to identify features on the plain film that will help to predict the likely outcome of the attack as the severity of the illness and the likelihood of perforation often preclude more elaborate radiological procedures at this stage.

Previous studies have shown that patients with persistent distension of small bowel loops are particularly prone to develop toxic megacolon. ${ }^{45}$ In these studies, the areas of intestinal gas shadows were measured by means of a planimeter.

In our study, we defined small bowel distension as the presence of three or more gas filled loops of small bowel. This can be easily assessed by clinicians and does not require the use of any mechanical device. We found that the presence of small bowel distension on a plain abdominal radiograph in a patient with severe ulcerative colitis may predict a poor response to medical treatment as a significantly higher proportion of patients who required colectomy had distension of their small bowel on plain films when compared with the group that responded favourably to medical therapy.

The mechanisms leading to the small intestinal accumulation of gas in some patients with severe ulcerative colitis are unclear. In ulcerative colitis, the small bowel is unaffected by the disease and hence the mechanisms that may account for toxic colonic dilatation such as severe inflammation with destruction of the myenteric plexus cannot be implicated. We found that the mean serum potassium concentration of patients who had small bowel distension was not significantly different from that of patients who did not have small bowel distension. Therefore, ileus as a result of hypokalaemia was unlikely to be the main explanation of small bowel gas accumulation. It is, however, likely that a number of factors alone or in combination such as hypocalcaemia, hypomagnessemia, and metabolic alkalosis may have resulted in reduced propulsive activity of the intestine and hence small bowel gaseous distension.

The presence of mucosal islands on plain radiographs of the abdomen in patients with severe ulcerative colitis may be an indication of impending dilatation and disintegration and has been associated with a poor clinical outcome. ${ }^{9}$ This is supported by our study. Eight of 10 patients $(80 \%)$, who had mucosal islands on their plain abdominal films required colectomy. Of these 10 patients, nine also had evidence of small bowel distension. Thus, mucosal islands are seen much less frequently than small bowel dilatation but, when present, are indicative of very severe disease.

There were only three cases of toxic megacolon, which is too few for us to make any reasonable conclusions as to whether small bowel distension predicts a tendency for the development of toxic megacolon. This small number may also reflect the Units policy for early surgery in patients who are severely ill and who fail to respond favourably to intensive medical therapy.

In conclusion, the presence of small bowel distension on the plain abdominal radiographs of patients with severe ulcerative colitis is associated with a poor response to medical therapy.

1 Wolf BS, Marshak RH. Toxic segmental dilatation of the colon during the course of fulminating ulcerative colitis; roentgen findings. Am F Roentgenol 1959; 82: 985-95.

2 Rice BP. Plain abdominal film roentgenographic diagnosis of ulcerative diseases of the colon. Am $\mathcal{F}$ Roentgenol 1968; 104 544-50.

3 McConnell F, Hanelin J, Robbins LL. Plain film diagnosis of fulminating ulcerative colitis. Radiology 1958; 71: 674-82.

4 Vernia P, Colaneri O, Tomei E, et al. Intestinal gas in ulcerative colitis. Dis Colon Rectum 1979; 22: 346-9.
col

5 Caprilli R, Vernia P, Latella G, Torsoli A. Early recognition of Caprilli R, Vernia $P$, Latella G, Torsoli A. Early recogn
toxic megacolon. $\mathcal{f}$ Clin Gastroenterol $1987 ; 9: 160-4$.

6 Truelove SC, Witts LJ. Cortisone in ulcerative colitis. Final report on a therapeutic trial. BMF 1955; 2: 1041-8.

7 Bartram CI. Ulcerative colitis. In: Radiology in inflammator bowel disease. New York: Marcel Decker, 1983: 31-61.

8 Hywel-Jones J, Chapman M. Definition of megacolon in colitis Gut 1969; 10: 562-4.

9 Bryan NB, Phillida AS. An indication for surgery in acute ulcerative colitis. Lancet 1964; ii: 1272-3. 